\title{
Les tendances au sein des effectifs de la SCPH : réussites et enjeux
}

\author{
par Bruce Millin
}

$\mathrm{E}_{\mathrm{p}}^{\mathrm{n}}$

novembre 2013, j’ai assisté au sommet sur la gouvernance présenté par la Société canadienne des directeurs d'association. L'événement réunissait des membres de directions d'associations de partout au pays qui se sont penchés, d'une part, sur les défis auxquels sont confrontées des associations comme la Société canadienne des pharmaciens d'hôpitaux $(\mathrm{SCPH})$ et, d'autre part, sur les occasions que celles-ci peuvent saisir. Voici quelques-unes des principales tendances observées au sein de l'infrastructure des associations :

- les membres ne voient pas l'intérêt de faire partie d'une association;

- la croissance accélérée du nombre de membres qui quittent après une première évaluation de la valeur qu'une association offre;

- la stagnation ou la réduction des effectifs.

L'été dernier, la SCPH a effectué un exercice de réflexion dans le but de vérifier si ces tendances traduisaient la situation de la Société. Voici un résumé de ce que nous avons appris en examinant les caractéristiques démographiques de nos effectifs entre les années 2008-2009 et 2012-2013.

La constatation la plus encourageante est le nombre croissant de membres de la SCPH! Le nombre total de membres en 2012-2013 a atteint 3247, une augmentation de $5 \%$ par rapport à l'année précédente. En fait, le nombre de pharmaciens qui sont membres de la SCPH a touché un sommet en cinq ans lors de la plus récente année d'adhésion. Le nombre de membres étudiants a aussi augmenté pour atteindre son plus haut niveau en cinq ans en 2012-2013, avec environ 100 membres de plus que les années précédentes. D'ailleurs, les bonnes nouvelles s'accumulent dans la catégorie des nouveaux membres. Après quelques années qui ont vu le nombre de nouveaux membres diminuer, 2012-2013 a été marquée par une augmentation du nombre de personnes adhérant pour la première fois à la SCPH pour ainsi retrouver les mêmes chiffres qu'en 2008-2009. Donc, en ce qui a trait aux effectifs, la tendance est positive. La SCPH attire plus de membres, entre autres plus de nouveaux venus dans la profession. La question suivante de l'exercice était : sommes-nous en mesure de conserver nos membres?

La SCPH a scruté trois mesures importantes relatives à ses effectifs : le taux de fidélisation, le taux de perte et la période de roulement. Elles illustrent dans quelle mesure la SCPH arrive à

conserver les membres qu'elle a acquis. La fidélisation est un point fort de la Société qui conserve toujours au moins $75 \%$ de ses membres d'une année à l'autre et, ainsi, en perd moins de $25 \%$. À un taux de perte de 20 à $25 \%$, la période de roulement des effectifs est de quatre à cinq ans; en d'autres termes, les effectifs de la SCPH disparaitraient complètement en quatre à cinq ans sans recrutement de nouveaux membres. Toutefois la $\mathrm{SCPH}$ a jusqu’à présent réussi à attirer suffisamment de nouveaux membres pour surcompenser les pertes.

Ces mesures montrent aussi que les deux premières années d'adhésion, au cours desquelles les membres jugent si la SCPH a quelque chose de valeur à leur offrir, sont les plus décisives. À cet égard, le taux de fidélisation des membres actifs qui renouvellent leur adhésion après leur deuxième année est de $69 \%$, un excellent chiffre. En comparaison, le taux de fidélisation tombe à $60 \%$ pour les pharmaciens membres qui en sont à leur première année d'adhésion, et à $35 \%$ pour les résidents en pharmacie et les étudiants diplômés qui passent de la catégorie d'adhésion " en formation " à " actif ". Il est nécessaire de déployer plus d'énergie pour faire en sorte que ces groupes cibles découvrent la valeur de l'adhésion à la SCPH.

Comme je l'ai appris au sommet sur la gouvernance, les associations prospères $\mathrm{du} 21^{\mathrm{e}}$ siècle sont celles qui auront concentré leurs énergies sur la valeur qu'elles offrent à leurs membres. La SCPH est une organisation influente qui soutient les efforts de ses membres pour développer et rechercher l'excellence dans les établissements de santé et dans d'autres milieux de soins de santé en collaboration, ce que confirment nos effectifs croissants. Néanmoins l'aptitude à long terme de la Société à guider la pratique sera compromise si nous ne gagnons pas la fidélité des pharmaciens d'établissements. Pour paraphraser Albert Einstein, n'essayons pas de devenir une association qui a du succès, mais essayons plutôt de devenir une association qui a de la valeur.

Bruce Millin, B. Sc. (Pharm.), ACPR, est président désigné et agent de liaison interne pour la Société canadienne des pharmaciens d'hôpitaux.

Remerciements : J'aimerais remercier Emma Attfield, interne à la SCPH pendant l'été 2013, qui a rassemblé les données apparaissant dans le présent document. 